\title{
Nonalcoholic fatty liver disease proven by transient elastography in patients with coronary heart disease
}

\author{
Ivana Mikolaševićc ${ }^{1 *}$, Lidjja Orlić1, Sandra Milić', Vesna Lukenda², Ervin Avdović1, Sanjin Rački', \\ Davor Štimac', Luka Zaputović ${ }^{1}$ \\ ${ }^{1}$ University of Rijeka School of Medicine, University Hospital Centre Rijeka, Rijeka, Croatia \\ 'General Hospital "Dr. Josip Benčević", Slavonski Brod, Croatia
}

\begin{abstract}
Objectives: The current importance of non-alcoholic fatty liver disease (NAFLD) and its link to the metabolic syndrome (MS) has encouraged interest in its possible role in the development of atherosclerosis. Recent studies have shown that NAFLD is not only a marker of cardiovascular disease (CVD), but may be actively involved in its pathogenesis. Nevertheless, the relationship between NAFLD and coronary heart disease (CHD) is still poorly understood. Therefore, we evaluated how frequently NAFLD is present in patients with $\mathrm{CHD}$. In this cross-sectional study we assessed the frequency of NAFLD in the above mentioned population by using transient elastography (TE). To our knowledge this is the first study using TE in assessment of NAFLD in CHD patients.
\end{abstract}

Patients and Methods: Sixty patients with proven CHD were enrolled. Controlled attenuation parameter (CAP) was used to detect and quantify liver steatosis by using TE (Fibroscan $^{\circledast}$, Echosense, Paris, France). The cut-off value for defining liver steatosis was CAP $238 \mathrm{~dB} / \mathrm{m}$ and cut-off value for defining the presence of fibrosis was liver stiffness of $>7 \mathrm{kPa}$. By CAP being implemented on TE, both liver steatosis and fibrosis can be evaluated simultaneously. Accordingly, NAFLD was defined by the presence of steatosis with CAP values $238 \mathrm{~dB} / \mathrm{m}$ regardless of presence or absence of any stage of fibrosis.

Results: Of the 60 patients, 39 (65\%) had CAP >238 dB/m and by definition NAFLD. According to the literature the grades of liver steatosis were defined by CAP values: 6 $(15.4 \%)$ patients had grade 1, $11(28.2 \%)$ had grade 2, 22 $(56.4 \%)$ had grade 3 . Among the patients with NAFLD, 15 patients $(38.5 \%)$ had in addition liver stiffness $>7 \mathrm{kPa}$. The severity of liver steatosis correlated positively with the total cholesterol, triglycerides, ALT and GGT levels, while there was negative correlation with hemoglobin and serum iron levels.

Conclusion: These results suggest that NAFLD proven by TE is highly prevalent in patients with CHD. Although the treatment of obesity, diabetes mellitus type 2 , hypertension and dyslipidemia could reduce NAFLD occurrence, its influence on CVD mortality is still not well established. Further prospective studies investigating this association are needed. The main finding of our study is that TE provides the opportunity of non-invasive screening for NAFLD in CHD patients, because it is a quick, simple, reliable and repeatable method and more cost-effective than liver biopsy.

KEYWORDS: non-alcoholic fatty liver disease, metabolic syndrome, cardiovascular disease, transient elastography.

CITATION: Cardiol Croat. 2013;8(9):315.

Received: $31^{\text {st }}$ Jul 2013

*Address for correspondence: Klinički bolnički centar Rijeka, Tome Strižića 3, HR51000 Rijeka, Croatia.

Phone: +385-51-407-487 Fax: +385-51-407-156

E-mail: ivana.mikolasevic@gmail.com

\section{Literature}

1. Gaggini M, Morelli M, Buzzigoli E, DeFronzo RA, Bugianesi E, Gastaldelli A. Non-alcoholic fatty liver disease (NAFLD) and its connection with insulin resistance, dyslipidemia, atherosclerosis and coronary heart disease. Nutrients. 2013;5(5):1544-60. 